\title{
COMPARACIÓN DEL MUESTREO DE ESPECIES QUÍMICAS EN ZONAS REACTIVAS MEDIANTE EL USO DE SONDA CON Y SIN CUELLO SÓNICO
}

\section{COMPARISON OF SAMPLING CHEMICAL SPECIES IN REACTING ZONES USING PROBES WITHOUT SONIC NOZZLE}

\author{
Francisco Cadavid $^{1} \quad$ Julián Uribe $^{1} \quad$ Andrés Amell $^{1}$ \\ Recibido 9 de marzo de 2009, aceptado 1 de junio de 2010 \\ Received: March 9, 2009 Accepted: June 1, 2010
}

\begin{abstract}
RESUMEN
El análisis de los productos de combustión en las zonas de reacción y al interior de cámaras de combustión requiere, con respecto a las medidas efectuadas en chimeneas, de equipos y de métodos particulares de muestreo. Así por ejemplo, en las zonas donde hay presencia de llama se deben utilizar dispositivos experimentales no intrusivos, tales como las técnicas láser, o intrusivos como las sondas de muestreo. El empleo de los primeros implica altos costos mientras que el de los segundos deben garantizar que las reacciones de combustión no continúen al interior de la sonda pues de lo contrario se producen errores importantes en la medición. En este trabajo se presenta una comparación de los resultados obtenidos al realizar muestreos al interior de la zona de reacción de una llama de premezcla parcial empleando dos tipos de sondas: una con cuello sónico y enfriamiento convectivo de la muestra y la otra constituida simplemente por un tubo en acero inoxidable para identificar si existen efectos térmicos y cinético-químicos que generen diferencias en la medición de las especies mayoritarias. Los resultados muestran que al emplear una sonda con un tubo liso se estarían presentando errores en las mediciones de $\mathrm{CO}_{2}$ y CO cercanos al 7,0\% y en las de $\mathrm{O}_{2}$ superiores al $14 \%$.
\end{abstract}

Palabras clave: Sondas de muestreo de gases, enfriamiento aerodinámico, sondas intrusitas.

\begin{abstract}
The analysis of combustion products inside flames reaction zones and combustion chambers requires special equipment and methods of sampling compared to measurements made in chimneys. For example, non-intrusive experimental devices, such as laser techniques, or intrusive ones, such as sampling probes, should be used in zones where a flame is present. The use of the first ones means high costs, while the second ones must guarantee that the combustion reactions do not continue inside the sample probe; otherwise significant errors occur in the species measurements. To identify whether there are thermal and chemical kinetic effects that produce differences in the major species measurement, this paper presents a comparison of sampling results inside the reaction zone of a partially premixed flame when two types of sample probes are used: one with a sonic neck and a convective cooling of the sample, and another made just of a straight stainless steel tube. The results show that when using a straight tube there are errors in $\mathrm{CO}_{2}$ and $\mathrm{CO}$ measurements up to 7.0\%, and errors in $\mathrm{O}_{2}$ measurements above $14 \%$.
\end{abstract}

Keywords: Gas sample probe, aerodynamic quenching, interference probes.

\section{INTRODUCCIÓN}

La determinación de las concentraciones locales de especies químicas estables, al interior de cámaras de combustión, en zonas tales como las llamas, donde aun se dan reacciones químicas, permite comprender mejor la forma como se dan las reacciones, así como manejar mejor los parámetros de combustión tales como la relación aire/ combustible y la calidad del mezclado. Con el conocimiento de estas concentraciones se busca limitar la producción de sustancias contaminantes, mejorar la estabilidad de la llama y comprender la distribución de los fluxes de calor, para manejar mejor los intercambios de calor.

Durante los pasados treinta años las investigaciones en combustión han logrado avances significativos como

1 Grupo de Ciencia y Tecnología del Gas y Uso Racional de la Energía-GASURE. Facultad de Ingeniería. Universidad de Antioquia. Calle 67 \# 53-108. Medellín, Colombia. E-mail: fcadavid@udea.edu.co 
resultado del desarrollo de técnicas de medición para flujos reactivos tanto a nivel de laboratorio como en instalaciones de combustión industriales [1-4].

Aunque hay disponibles numerosas técnicas de diagnóstico, la medición de las cantidades escalares de un fluido reactivo, esto es, temperatura y concentración de especies estables $\left(\mathrm{CO}, \mathrm{CO}_{2}, \mathrm{O}_{2}, \mathrm{NO}\right.$, etc.), se ha basado tradicionalmente en la introducción de sondas dentro del flujo [3 - 8]. Sin embargo, al ser utilizadas siempre surge la siguiente pregunta: ¿Si al introducir una sonda dentro de un flujo de fluido este se ve perturbado, son entonces fiables las mediciones realizadas? [9].

En respuesta a lo anterior, se han venido desarrollando técnicas ópticas no intrusivas, basadas en la espectrometría láser $[1,2]$. No obstante lo anterior, son técnicas que se caracterizan porque su puesta a punto es muy compleja y muy costosa. Asimismo, requieren de accesos ópticos con vidrios especiales que, además de resistir las altas temperaturas, deben garantizar una mínima distorsión del rayo láser. Además, con las técnicas láser es difícil obtener datos de alta calidad en sistemas de combustión con llamas altamente luminosas y con presencia de material particulado [2].

Lo anterior explica por qué las sondas de muestreo siguen siendo aplicadas en la medición de cantidades escalares, lo cual representa un incentivo para continuar refinando estas técnicas en aras de incrementar su precisión y de expandir su aplicabilidad [5]. Además, las sondas de muestreo de gases son las que se utilizan en el estudio detallado de las llamas, en particular cuando se busca obtener el perfil de especies de hidrocarburos relevantes en la formación de aromáticos y son también ampliamente utilizadas en el estudio de llamas ricas en combustible [9-10].

La utilización de las sondas de muestreo de gases, en zonas en donde se dan reacciones químicas, implica primero que todo su adecuado dimensionamiento y enfriamiento así como el enfriamiento rápido de la muestra para evitar que las reacciones químicas de los productos estables continúen al interior de la sonda. En el presente trabajo se realiza una breve descripción del principio de funcionamiento de las sondas de muestreo de gases que funcionan bajo el principio del enfriamiento aerodinámico, asimismo se presenta una comparación del muestreo de especies químicas en zonas reactivas utilizando dos tipos de sondas: una con cuello sónico y otra sin cuello sónico con el propósito de identificar si existen interferencias térmicas y cinéticoquímicas que generen diferencias en la medición de las especies mayoritarias $\left(\mathrm{CO}_{2}, \mathrm{CO}\right.$ y $\left.\mathrm{O}_{2}\right)$.

\section{TIPOS DE SONDAS DE MUESTREO}

Aunque existen muchos tipos de sondas de muestreo, su diseño se ha basado, en general, en el enfriamiento convectivo o en técnicas de enfriamiento aerodinámico. Un primer grupo de sondas incluye las sondas en acero inoxidable del tipo pitot [11]. Otro tipo de sondas son las que aplican el enfriamiento convectivo en las cuales, para asegurar un rápido enfriamiento, la muestra se enfría directamente con agua. Luego se deben separar la muestra y el agua para así evitar que entre agua al sistema de análisis de productos de combustión [12]. El tercer grupo de sondas emplean el enfriamiento aerodinámico realizando una rápida expansión cerca al extremo de la sonda. Estas sondas tienen la ventaja de reducir la interferencia, pero pueden tener bajas tasas de enfriamiento, por lo que se combina la expansión aerodinámica y el enfriamiento convectivo [13]. Son este tipo de sondas las que son tratadas en este documento y a partir de aquí se les denominará sonda sónica.

\section{PRINCIPIO DE FUNCIONAMIENTO DE LAS SONDAS SÓNICAS}

Las sondas para la toma de las muestras de humos por aspiración toman la muestra a la temperatura del medio, y las transportan al analizador de gases. La temperatura a la que se encuentran los gases posibilita que las reacciones químicas se sigan presentando en el trayecto hacia el analizador, lo que puede generar errores en las mediciones, ya que la composición de los humos en el punto de toma de la muestra va ser diferente a la composición indicada por el equipo de análisis de gases.

Las sondas con cuello sónico generan un enfriamiento brusco de la muestra a la entrada de la sonda. Los gradientes de temperatura y presión son muy fuertes en la zona del cuello sónico, lo cual evita que las reacciones se puedan seguir dando en el trayecto hacia el analizador.

Para que una sonda de muestreo genere un enfriamiento aerodinámico de la muestra de gases de combustión se requiere que el flujo de gases aspirado por la sonda sea acelerado hasta lograr condiciones supersónicas, garantizando números de Mach mayores de 2. Con esto se busca, vía la expansión geométrica de la sonda, reducir la temperatura de la muestra de gases. La expansión debe ser lo suficientemente grande para así garantizar una disminución de la temperatura de la muestra a una tasa del orden de $10^{8} \mathrm{~K} / \mathrm{seg}$ [11]. La sonda también debe tener una zona de sección transversal constante, la cual debe ubicarse inmediatamente después del extremo por donde se aspira la muestra, en la que, a través de un enfriamiento convectivo con una sustancia refrigerante, se logre una disminución adicional de la temperatura de 
la muestra. En la Figura 1 se presenta una representación esquemática de este tipo de sondas con las diferentes partes que la componen.

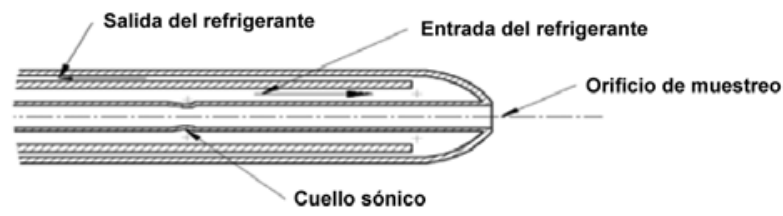

Figura 1. Vista en corte de sonda con cuello sónico y enfriamiento convectivo.

\section{Descripción del banco de análisis de los gases}

La evaluación experimental de la sonda se realizó en un banco de análisis de gases de combustión, mostrado en la Figura 2 y constituido por los siguientes elementos:

- Sonda: en este trabajo se emplean dos sondas, con cuello sónico y refrigerada por agua con un orificio de muestreo de $1,58 \mathrm{~mm}$ de diámetro externo y un tubo liso de 6,34 $\mathrm{mm}$ de diámetro externo.

- Unidad de acondicionamiento de gases, marca M \& C, modelo PSS-5. Esta unidad es utilizada para succionar los gases de combustión desde la cámara de combustión para luego inyectarlos al analizador de gases. Asimismo, con ésta también se condensa el vapor de agua presente en los productos de combustión, al enfriar la muestra por debajo de $\operatorname{los} 5{ }^{\circ} \mathrm{C}$, con lo cual se evitan daños en el analizador.

- Analizador de gases de combustión, marca SICKMAIHAK, modelo S710. Este equipo emplea módulos infrarrojos no dispersivos (NDIR por sus siglas en inglés) para cuantificar la concentración volumétrica de monóxido de carbono (CO) y dióxido de carbono $\left(\mathrm{CO}_{2}\right)$. También tiene un módulo paramagnético para cuantificar las concentraciones volumétricas de oxígeno $\left(\mathrm{O}_{2}\right)$.

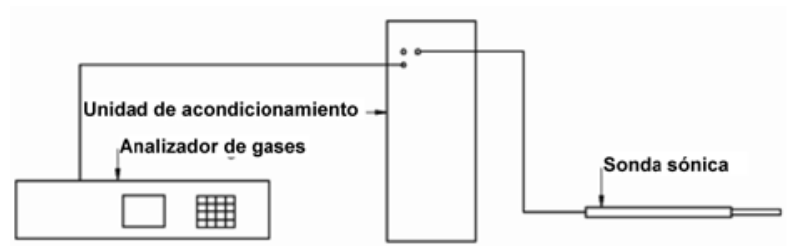

Figura 2. Banco de muestreo.

Descripción del montaje experimental para la extracción y el análisis de los gases

En la Figura 3 se muestra el montaje experimental empleado. Está constituido por un quemador atmosférico de premezcla parcial a gas natural, un dispositivo para ubicar los dos tipos de sondas empleadas durante los ensayos. Los productos de combustión producidos en la zona de reacción de la llama son aspirados por el analizador de gases, en el que se pueden cuantificar las emisiones de $\mathrm{CO}_{2}$ (0-25\%), CO (0-60.000 ppm) y $\mathrm{O}_{2}(0-100 \%)$. El caudal de agua utilizado para realizar el enfriamiento convectivo es de $8,45 \mathrm{ml} / \mathrm{min}$ y el caudal de muestra aspirado por el analizador es de $150 \mathrm{Nl} / \mathrm{h}$.

Las muestras de productos de combustión se tomaron en tres puntos ubicados a $20 \mathrm{~mm}, 40 \mathrm{~mm}$ y $60 \mathrm{~mm}$ de la cabeza del quemador (ver Figura 3). En cada punto de medición se realizaron cinco réplicas. Los resultados presentados en la Figura 4 corresponden a los valores promedio de las cinco réplicas realizadas. La desviación estándar promedio para las mediciones realizadas con la sonda sin cuello sónico fue de $0,29 \%$ de $\mathrm{CO}_{2}, 980 \mathrm{ppm}$ de CO y $0,09 \%$ de $\mathrm{O}_{2}$. Para la sonda con cuello sónico ésta fue de $0,12 \%$ de $\mathrm{CO}_{2}, 515$ ppm de $\mathrm{CO}$ y $0,06 \%$ de $\mathrm{O}_{2}$.

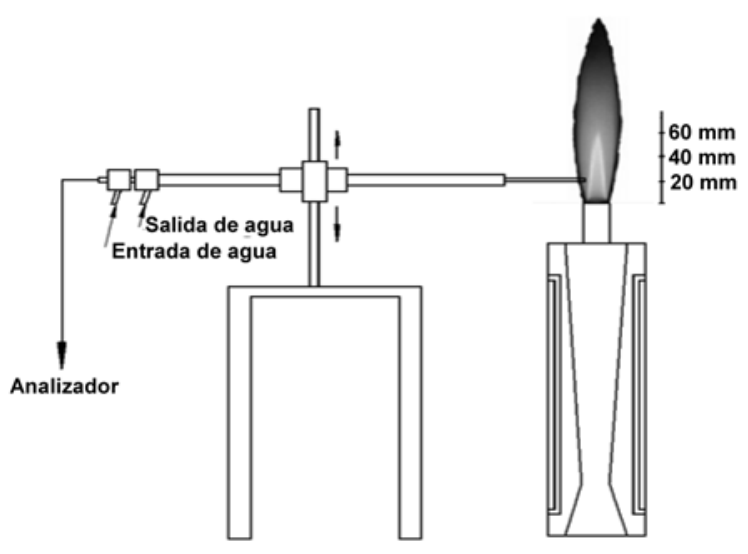

Figura 3. Montaje experimental.

Para obtener resultados fiables de emisiones en la zona de reacción es importante que la sonda empleada para la toma de muestras garantice que las reacciones de combustión no continúen en el trayecto hasta el analizador. Si esto ocurre las emisiones de $\mathrm{CO}_{2}$ serán sobrestimadas, mientras que las emisiones de $\mathrm{CO}_{2} \mathrm{O}_{2}$ se verán subestimadas. Esto se da porque el $\mathrm{CO}$, por ser aspirado a altas temperaturas, reacciona con el $\mathrm{O}_{2}$ y con radicales $\mathrm{OH}$ para seguir formando $\mathrm{CO}_{2}$.

\section{RESULTADOS Y ANÁLISIS}

En la Figura 4 se presentan las emisiones de $\mathrm{CO}_{2}, \mathrm{CO}$ y $\mathrm{O}_{2}$ obtenidas al emplear dos tipos de sondas de muestreo: una constituida por un tubo liso en acero inoxidable 304 de $6,34 \mathrm{~mm}$ de diámetro externo y la otra fabricada con tubos de acero inoxidable 304 con un cuello sónico 
de 1,0 mm de diámetro interno y además refrigerada convectivamente por agua. Con esta última se busca producir un enfriamiento rápido de la muestra para evitar que las reacciones de combustión continúen en el trayecto hacia el analizador de gases. De los resultados obtenidos con los dos tipos de sondas empleadas se tiene que al emplear un tubo liso, las emisiones de $\mathrm{CO}_{\mathrm{y}} \mathrm{O}_{2}$ son respectivamente, en promedio, 2.615 ppm $(0,26 \%)$ y 3.100 ppm $(0,31 \%)$ mayores que las obtenidas con la sonda con cuello sónico y enfriamiento convectivo; mientras que las emisiones de $\mathrm{CO}_{2}$ son, en promedio, 5.600 ppm $(0,56 \%)$ menores al emplear la sonda sónica.

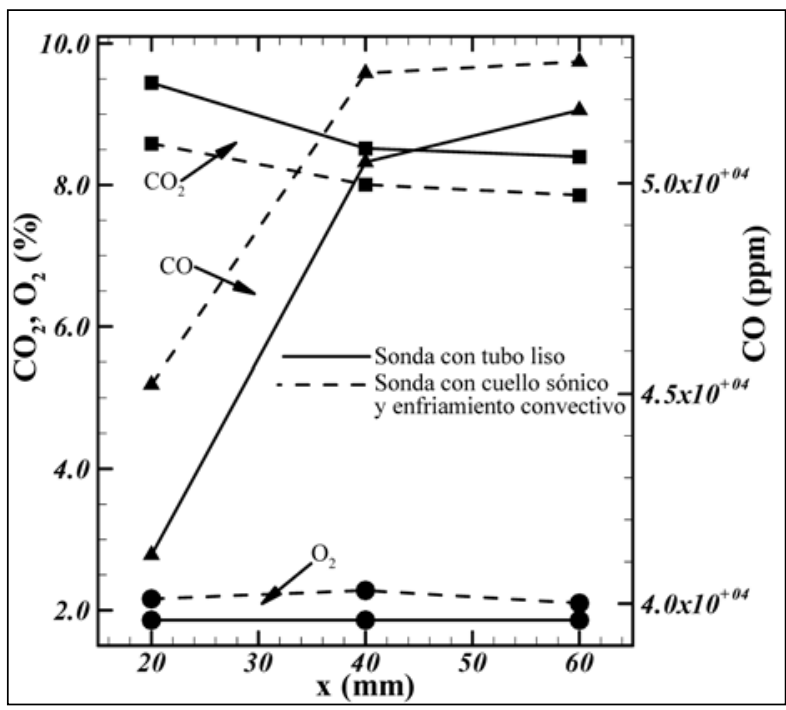

Figura 4. Emisiones de especies mayoritarias en la zona de reacción de una llama empleando, como sondas de medición, un tubo liso y una sonda con cuello sónico y enfriamiento convectivo. ․: $\mathrm{CO}_{2} ; \mathbf{\Lambda}: \mathrm{CO} ; \mathbf{O}: \mathrm{O}_{2}$.

El comportamiento de las dos sondas empleadas muestra cómo, al realizar muestreos con tubos lisos, en zonas del sistema de combustión donde aun hay reacciones químicas, se generan errores de medición importantes y por lo tanto posibles interpretaciones erróneas sobre el comportamiento del sistema de combustión. Por ejemplo, si asumimos que los valores de las emisiones obtenidos empleando la sonda sónica son los verdaderos, se estarían presentando errores en las mediciones de $\mathrm{CO}_{2}$ y $\mathrm{CO}$ cercanos al 7,0\% y en el $\mathrm{O}_{2}$ superior al $14 \%$.

\section{CONCLUSIONES}

El control de las emisiones contaminantes en sistemas de combustión siempre se debe realizar, debido a que existen normas gubernamentales que las regulan pues afectan a los seres humanos, a los animales y al ambiente en general. Es por lo anterior que históricamente se han desarrollado sistemas de muestreo que permitan estudiar los fenómenos de combustión en las zonas de reacción y así poder conceptuar sobre las posibles formas de evitar la formación dichas sustancias contaminantes. Uno de los métodos utilizados para cuantificar dichas especies es vía la utilización de sondas de muestreo. En este trabajo se estudió el funcionamiento de dos tipos de sondas, una constituida por un tubo liso y la otra que emplea dos formas de enfriamiento de la muestra: el enfriamiento aerodinámico más enfriamiento por convección.

En la sonda con cuello sónico y enfriamiento convectivo se comprobó que las reacciones de combustión no continuaban en el trayecto hacia el analizador. Al emplear una sonda sin cuello sónico y sin enfriamiento convectivo se presentan errores en las mediciones de $\mathrm{CO}_{2}$ y $\mathrm{CO}$ cercanos al 7,0\% $\mathrm{y}$ en el $\mathrm{O}_{2}$ superiores al $14 \%$.

\section{AGRADECIMIENTOS}

Los autores desean expresar sus más sinceros agradecimientos a COLCIENCIAS por la financiación y apoyo en la ejecución del proyecto, bajo contrato 1115-332-18837, que dio origen a los resultados aquí planteados.

\section{REFERENCIAS}

[1] A.C. Eckbreth. "Laser Diagnostics for Combustion Temperature and Species". Combustion Science and Technology Book Series. Vol. 3. 1988.

[2] M. Linne. "Spectroscopic measurement: An introduction to the fundamentals". Academic Press. London, UK. 2002.

[3] N. Chigier. "Combustion measurements". Hemisphere. 1991.

[4] W.P. Jones and J.H. Whitelaw. "Modelling and measurements in turbulent combustion". Twentieth Symposium (International) on Combustion. Vol. 20, Issue 1, pp. 233-249. 1985.

[5] M.V. Heitor and A.L.N. Moreira. "Thermocouples and sample probes for combustion studies". Progress in Energy and Combustion Science. Vol. 19, Issue 3, pp. 259-278. 1993.

[6] I.V. Dyakov, J. De Ruyck and A.A. Konnov. "Probe sampling measurements and modeling of nitric oxide formation in ethane + air flames". Fuel. Vol. 86, Issues 1-2, pp. 98-105. January, 2007. Doi:10.1016/j.fuel.2006.06.003

[7] M. Costa, C. Parente and A. Santos. "Nitrogen oxides emissions from buoyancy and momentum controlled turbulent methane jet diffusion flames". 
Experimental Thermal and Fluid Science. Vol. 28, Issue 7, pp. 729-734. September, 2004. Doi:10.1016/j.expthermflusci.2003.12.010

[8] T. Mahmud, S.K. Sangha, M. Costa and A. Santos. "Experimental and computational study of a lifted, non-premixed turbulent free jet flame". Fuel. Vol. 86, Issue 5-6, pp. 793-806. March-April, 2007. Doi:10.1016/j.fuel.2006.08.030

[9] A.T. Hartlieb, B. Atakan and K. Kohse-Höinghaus. "Effects of a Sampling Quartz Nozzle on the Flame Structure of a Fuel-Rich Low-Pressure Propene Flame". Combustion and Flame. Vol. 121, Issue 4, pp. 610-624. June, 2000. Doi:10.1016/S00102180(99)00176-5

[10] C.S. McEnally, L.D. Pfefferle, B. Atakan and K. Kohse-Höinghaus. "Studies of aromatic hydrocarbon formation mechanisms in flames: Progress towards closing the fuel gap" Progress in Energy and Combustion Science. Vol. 32, Issue 3, pp. 247-294. 2006. Doi:10.1016/j.pecs.2005.11.003.

[11] A.N. Hayhurst and N.R. Telford. "Mass spectrometric sampling of ions from atmospheric pressure flames I: Characteristics and calibration of the sampling system". Combustion and Flame. Vol. 28, pp. 67-80. 1977. Doi:10.1016/0010-2180(77)90009-8

[12] M.M. Hassan, F.C. Lockwood and H.A. Moneib. "Measurements in a gas-fired cylindrical furnace". Combustion Flame. Vol. 51, pp. 249-261. 1983. Doi:10.1016/0010-2180(83)90104-9

[13] M.B. Colket, L. Chiappetta, R.N. Guile, M.F. Zabielski and D.J. Seery. "Internal Aerodynamics of Gas Sampling Probes". Combustion and Flame. Vol. 44, Issues 1-3, pp. 3-14. 1982. Doi:10.1016/0010-2180(82)90058-X 